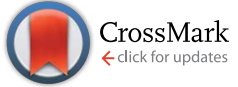

Cite this: RSC Adv., 2017, 7, 2637

Received 17th November 2016 Accepted 13th December 2016

DOI: 10.1039/c6ra26937k

www.rsc.org/advances

\section{Urchin-like CoO-C micro/nano hierarchical structures as high performance anode materials for Li-ion batteries $\uparrow$}

\begin{abstract}
Lili Liu, ${ }^{a}$ Lihui Mou, ${ }^{a}$ Jia Yu ${ }^{\text {bc }}$ and Shimou Chen*b
In this work, urchin-like microspheres consisting of radial carbon-coated cobalt monoxide nanowires are designed, to fabricate a micro/nano hierarchical structure for efficient Li-storage. A sequential conversion process is adopted, involving hydrothermal synthesis of an urchin-like cobalt carbonate hydroxide precursor, glycosidation of glucose as a coating layer, and calcination in an inert atmosphere to form a $\mathrm{CoO}-\mathrm{C}$ composite. On the one hand, the $\mathrm{CoO}$ framework provides a high Li-storage capacity with short transport paths and ordered free spaces. On the other hand, the carbon coating layer buffers the volume expansion of inner active materials during lithiation, while providing a stabilized electrode/electrolyte interface and rapid electronic conduction. When tested as an anode material for lithium ion batteries, the urchin-like $\mathrm{CoO}-\mathrm{C}$ delivers a reversible capacity of $755 \mathrm{~mA} \mathrm{~h} \mathrm{~g}^{-1}$ after 100 cycles. With rate increasing, the performance advantage of the $\mathrm{CoO}-\mathrm{C}$ composite electrode over a single $\mathrm{CoO}$ electrode is obvious.
\end{abstract}

\section{Introduction}

One key factor that has impeded the improvement of lithium ion batteries (LIBs) is the limited performance of the present electrode materials, which hardly satisfies the increasing demand of high energy \& power density and long-term stability. Fabricating composite nanostructure electrodes becomes a frontier issue due to the synergistic effect imparted by multiple compositions. ${ }^{1-3}$ As promising next-generation anode materials, transition metal oxides $\left(\mathrm{MO}_{x}, \mathrm{M}=\mathrm{Co}, \mathrm{Ni}, \mathrm{Cu}\right.$, etc.) exhibit high theoretical capacities, because of their unique conversion mechanism $\left(\mathrm{MO}_{x}+2 x \mathrm{Li}^{+}+2 x \mathrm{e}^{-} \leftrightarrow \mathrm{M}+x \mathrm{Li}_{2} \mathrm{O}\right) \cdot{ }^{4-6}$ For example, cobalt monoxide (CoO) delivers a high theoretical capacity of $715 \mathrm{~mA} \mathrm{~h} \mathrm{~g}{ }^{-1}$, almost twice that of a commercial graphite anode. However, $\mathrm{CoO}$ also suffers from serious drawbacks as LIB anodes, such as large expansion during lithiation process, repeated formation of solid electrolyte interface (SEI) film and slow $\mathrm{Li}^{+}$and electron diffusion, thus leading to poor rate capability and cycling stability. ${ }^{4,7}$ Up to now, several strategies have been adopted to address above issues, among which compositing and nanostructuring are two main methods to improve Li-storage capability. ${ }^{8-11}$

\footnotetext{
${ }^{a}$ School of Science, Beijing Technology and Business University, Beijing 100048, China ${ }^{b}$ Beijing Key Laboratory of Ionic Liquid Clean Process, Key Laboratory of Green Process and Engineering, Institute of Process Engineering, Chinese Academy of Sciences, Beijing 100190, China. E-mail: chenshimou@ipe.ac.cn ${ }^{c}$ University of Chinese Academy of Sciences, Beijing 100049, China

$\dagger$ Electronic supplementary information (ESI) available: SEM images, TG curve, XRD pattern, Raman spectrum, coulombic efficiencies, performance comparison table. See DOI: 10.1039/c6ra26937k
}

On the one hand, electrochemically stable coatings layers like carbon, can prevent a direct contact between $\mathrm{CoO}$ and electrolyte, and improve the originally poor electric conduction. ${ }^{12,13}$ Moreover, carbon coating can also buffer the stress imparted by volume expansion of inner active materials during cycles, thus leading to improved rate capability and cycling performance. For example, nitrogen-doped carbon-wrapped porous $\mathrm{CoO}$ nanocubes showed a reversible capacity of about $600 \mathrm{~mA} \mathrm{~h} \mathrm{~g}{ }^{-1}$ as LIB anode material. ${ }^{14}$ And carbon-coated CoO wall arrays on Ni foam also exhibited excellent lithium storage performance. ${ }^{15}$ Among them, thermal decomposition using lowcost glucose precursor is a well-established and applicable carbon coating method for large-scale production. ${ }^{16,17}$ On the other hand, fabricating 3D nanostructures with short transport paths and ordered free space is another effective strategy, such as nanoarrays, nanonets and nanowire clusters., ${ }^{70,11}$ For example, ultrathin $\mathrm{CoO}$ nanosheet arrays fabricated via galvanostatic electrodeposition achieved a capacity of about $1000 \mathrm{~mA} \mathrm{~h} \mathrm{~g}{ }^{-1}$ as a LIB anode. ${ }^{10}$ Particularly, microspheres composing of nanowires which fanned out, were able to relieve the volume variation stress during cycles, as well as provide a short ion transport pathway. As another cobalt-based oxide, pompon-like hierarchical $\mathrm{Co}_{3} \mathrm{O}_{4}$ microspheres were able to show superior Li-storage performance compared with nanoparticles and nanowires, proving great potential in LIB anode applications. ${ }^{18}$ Therefore, it is reasonable to combine 3D hierarchical microsphere architecture with carbon coating layer, for achieving further improved electrochemical performance.

In this work, an urchin-like CoO-C composite structure with micro/nano hierarchical structure was proposed and synthesized via a sequential conversion procedure (Scheme 1). 


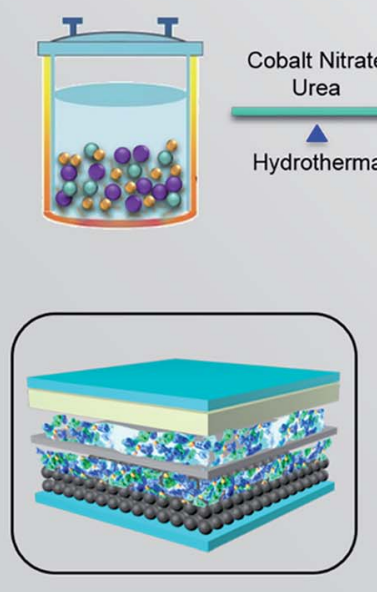

(a)

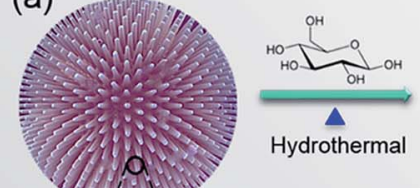

(b)

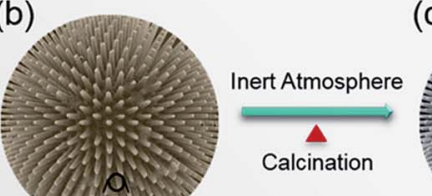

(c)

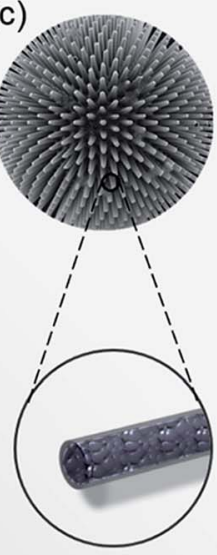

Glycosidation of Glucose
Cobalt(II) Oxide@Amorphous Carbon

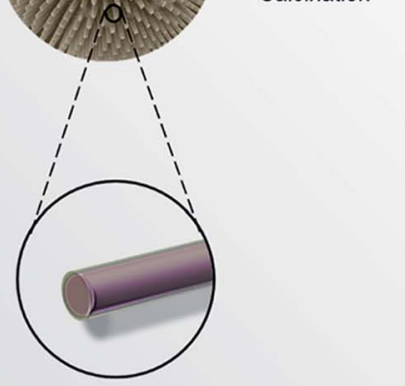

Scheme 1 The sequential conversion procedure for constructing urchin-like $\mathrm{CoO}-\mathrm{C}$ micro/nano hierarchical structure: (a) urchin-like cobalt carbonate hydroxide precursors prepared via one-pot hydrothermal synthesis. (b) Glycosidation of glucose on precursors via second hydrothermal synthesis. (c) Final $\mathrm{CoO}-\mathrm{C}$ prepared after calcination in inert atmosphere. (Left bottom: simulation diagram of the lithium ion battery with urchin-like $\mathrm{CoO}-\mathrm{C}$ as the anode).

Specifically, the preparation process included one-pot hydrothermal synthesis of urchin-like cobalt carbonate hydroxide precursors (Scheme 1a), subsequent coating process using glycosidation of glucose via second hydrothermal synthesis, and final calcination process in inert atmosphere to obtain $\mathrm{CoO}-\mathrm{C}$. The inner radial $\mathrm{CoO}$ nanowires can provide a high capacity and stable Li-storage framework, because of the conversion mechanism, short transport paths and ordered free space. Meanwhile, the external amorphous carbon coating layer provides stabilized electrode/electrolyte interface and enhanced electronic conduction and also alleviate the volume expansion of inner active materials during lithiation process. Therefore, such composite micro/nano hierarchical design was able to efficiently integrate advantages and circumvent drawbacks of the two different compositions, to form a synergistic Li storage system. ${ }^{19}$ It's worth noting that using glucose as the thermal decomposition precursor to fabricate the carbon coating layer is a low-cost and efficient technique, while other routines may suffer from a high cost (chemical vapor deposition or magnesiothermic reduction) or a poor electrochemical interface (ex situ mixing). When applied in LIB anode materials, benefiting from its unique compositional and structural characteristics, this synergistic nanostructured design has great potential in enhancing the Li-storage capability of transition metal oxides, especially for high rate charge/discharge.

\section{Experimental}

\section{Preparation methods}

All of the chemicals were analytical grade and were used without further purification. During a typical experiment, firstly $20 \mathrm{mmol}$ of cobalt nitrate hexahydrate $\left(\mathrm{Co}\left(\mathrm{NO}_{3}\right)_{2} \cdot 6 \mathrm{H}_{2} \mathrm{O}\right)$ and 5 mmole of urea $\left(\mathrm{CO}\left(\mathrm{NH}_{2}\right)_{2}\right)$ were dissolved in $40 \mathrm{~mL}$ of deionized water, and then the solution was transferred into a $50 \mathrm{~mL}$ Teflon-lined autoclave. After $5 \mathrm{~h}$ of hydrothermal reaction at $180^{\circ} \mathrm{C}$, the obtained hydrothermal products was rinsed by deionized water and ethanol for several times, and then dried at $80{ }^{\circ} \mathrm{C}$. Subsequently, $0.2 \mathrm{~g}$ of above hydrothermal product powders and $2 \mathrm{mmol}$ of glucose (or $4 \mathrm{mmol}$ for comparison) were heated under $180^{\circ} \mathrm{C}$ for $3 \mathrm{~h}$ (or $8 \mathrm{~h}$ for comparison) to conduct second hydrothermal process. Finally, after rinsing and drying, products of the second hydrothermal reaction were calcined at $500^{\circ} \mathrm{C}$ for $3 \mathrm{~h}$ in nitrogen atmosphere, to obtain the urchin-like $\mathrm{CoO}-\mathrm{C}$ micro/nano hierarchical composite. As a contrast, single $\mathrm{CoO}$ microspheres were prepared under similar reaction system and conditions, except that the second hydrothermal process was omitted.

\section{Characterizations}

Scanning electron microscopy (SEM) and high resolution scanning electron microscope (HRSEM) observations were carried out on the SU8020 microscope (Hitachi). Energy dispersive spectroscopy (EDS) analyses were performed using an Oxford X-MaxN attached to the Hitachi SU8020 at $20.0 \mathrm{kV}$, with a silicon slice as the substrate. Transmission electron microscopy (TEM) and high resolution transmission electron microscope (HRTEM) observations were performed with the JEM-2100F microscope (JEOL). Raman spectra were recorded using the LabRAM HR800 $514 \mathrm{~nm}$ Ar-ion laser (Horiba Jobin-Yvon). X-ray diffraction (XRD) patterns were recorded using a Bruker D8 Focus X-ray diffractometer equipped with a Ni-filtered $\mathrm{Cu}-\mathrm{K} \alpha$ radiation $(\lambda=0.15406$ $\mathrm{nm}$ ) source. X-ray photoelectron spectroscopy (XPS) data were obtained with the ESCALAB 250Xi (Thermo Fisher Scientific) using a monochromated Al-K $\alpha$ X-ray source. Thermo gravimetric (TG) analysis was performed using the STA7200RV (Hitachi High-Tech), with nitrogen atmosphere and a heating rate of $5{ }^{\circ} \mathrm{C}$ $\min ^{-1}$. The specific surface area and pore volume were measured by the $\mathrm{N}_{2}$ adsorption/desorption isotherm method on a Quadrasorb SI-MP analyzer. 


\section{Electrochemical measurements}

Electrochemical measurements were performed at room temperature using coin-type half-cells. The $\mathrm{CoO}-\mathrm{C}$ (or $\mathrm{CoO}$ ), super $\mathrm{P}$ and polyvinylidene fluoride (PVDF) were mixed with a weight ratio of $80: 10: 10$ using 1-methyl-2-pyrrolidinone as the solvent, followed by pasted onto copper foil and dried at $80{ }^{\circ} \mathrm{C}$. The active material mass was determined according to the weight difference between the electrode piece and bare copper foil piece. The loading mass for each electrode piece was about $3.2 \mathrm{mg}$. Lithium foils were used as counter electrodes. And Celgard 2400 membranes were used to separate the working electrode and counter electrode. The electrolyte was $1 \mathrm{~mol} \mathrm{~L}^{-1}$ of $\mathrm{LiPF}_{6}$ in EC/DEC/DMC mixture (1:1:1 in weight). To cycle coin cells, they were discharged to $0.01 \mathrm{~V}\left(v s . \mathrm{Li} / \mathrm{Li}^{+}\right)$and charged to $3.0 \mathrm{~V}\left(v s . \mathrm{Li} / \mathrm{Li}^{+}\right)$on the LAND battery testing system. Cyclic voltammetry (CV) data was recorded on the Autolab (PGSTAT302N) electrochemical workstation, with a scanning rate of $0.1 \mathrm{mV} \mathrm{s}^{-1}$ and a voltage range of $0.01-3.0 \mathrm{~V}$. Electrochemical impedance spectroscopy (EIS) measurements were carried out on the ACM Gill-AC-4 electrochemical station.

\section{Results and discussion}

\section{Morphology characterizations}

The morphologies of precursors and final products were investigated by SEM and HRSEM, to observe the constructing process of micro/nano hierarchical structure. In Fig. 1a and S1 (ESI $\dagger$ ), cobalt carbonate hydroxide precursors obtained after the first hydrothermal process exhibited an uniform urchin-like microsphere structure with diameters of 5-10 $\mu \mathrm{m}$. The nanowire basic units fanned out from the core, exhibiting diameters of 20-40 nm with relatively smooth surface (Fig. 1b). After second hydrothermal and calcination processes, CoO-C microspheres maintained a consistent structure with precursors (Fig. 1c and $\mathrm{S} 2 \dagger$ ).

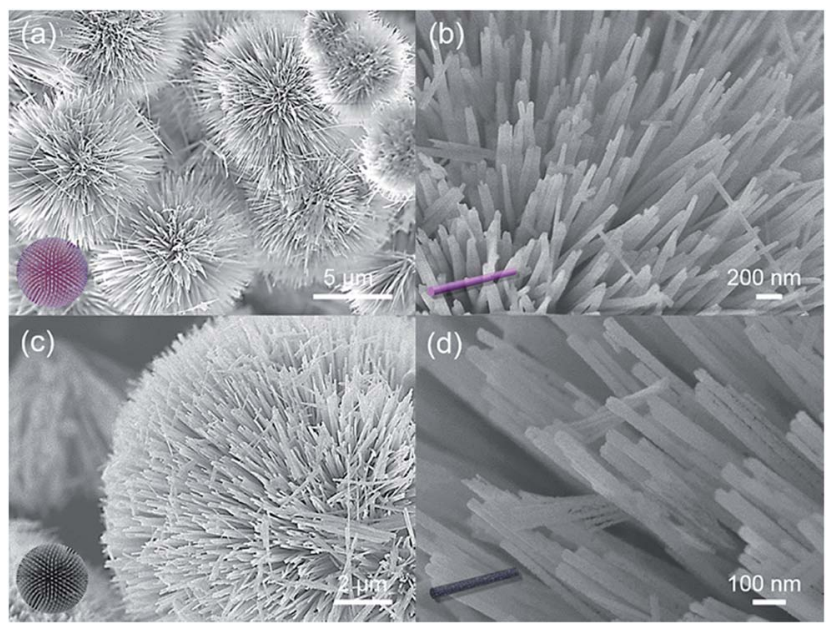

Fig. 1 Product morphologies at various synthesis stages: (a, and b) urchin-like cobalt carbonate hydroxide precursor microspheres, with diameters of $5-10 \mu \mathrm{m}$ and relatively smooth surface. (c and d) Final CoO-C microspheres, their radial nanowire basic units exhibiting relatively coarse surface. Insets: corresponding simulation diagrams.
The carbon-coating $\mathrm{CoO}$ nanowires acted as basic units to fabricate into an urchin-like microsphere, forming a unique micro/nano hierarchical structure. Meanwhile, it was observed that the radial nanowires exhibited slightly increased diameters of 30-50 nm, accompanying with relatively coarse surface due to calcination treatment (Fig. 1d). In addition, a more obvious carbon coating layer was observed when the glucose amount and reaction time of the second hydrothermal process were increased, because of the higher coating amount. However, the original urchin-like morphology could hardly be maintained, with the nanowire basic units becoming twisted and coarser (Fig. S3†).

The microstructure of the $\mathrm{CoO}-\mathrm{C}$ composite microsphere was further observed by TEM and HRTEM. Fig. $2 \mathrm{a}$ and $\mathrm{b}$ clearly demonstrated the unique micro/nano hierarchical structure. Fig. $2 c$ exhibited the porous structure of the nanowire basic unit, which might result from the gas releasing during calcination. ${ }^{20}$ This could provide better access for $\mathrm{Li}^{+}$, and shorten diffusion paths of $\mathrm{Li}^{+}$and electrons, thus benefiting the rate capability. ${ }^{21,22}$ In addition, a thin light-color coating layer was observed, which corresponded to the amorphous carbon coating layer. This might enable extra interfacial lithium storage, which was favorable to the energy density of this composite system. ${ }^{23}$ From the TEM observations (Fig. 2c and S4†), the thickness of the carbon coating layer was about 3-6 $\mathrm{nm}$. As shown in the HRTEM image of an individual CoO-C nanowire (Fig. 2d), two interplanar spacings of 0.21 and $0.25 \mathrm{~nm}$ were clearly observed, which resulted from $\mathrm{CoO}$ (200) and (111) lattice planes respectively, indicating the polycrystalline nature. ${ }^{24}$

\section{Compositional and structural characterizations}

The thermal behaviour of the precursor $\left(\mathrm{Co}\left(\mathrm{CO}_{3}\right)_{0.5}(\mathrm{OH})\right.$. $0.11 \mathrm{H}_{2} \mathrm{O}$ ) in nitrogen atmosphere was investigated by TG analysis (Fig. S5a $\dagger$ ). It was observed that a major thermal event took place at around $300{ }^{\circ} \mathrm{C}$ during the heating process. The total

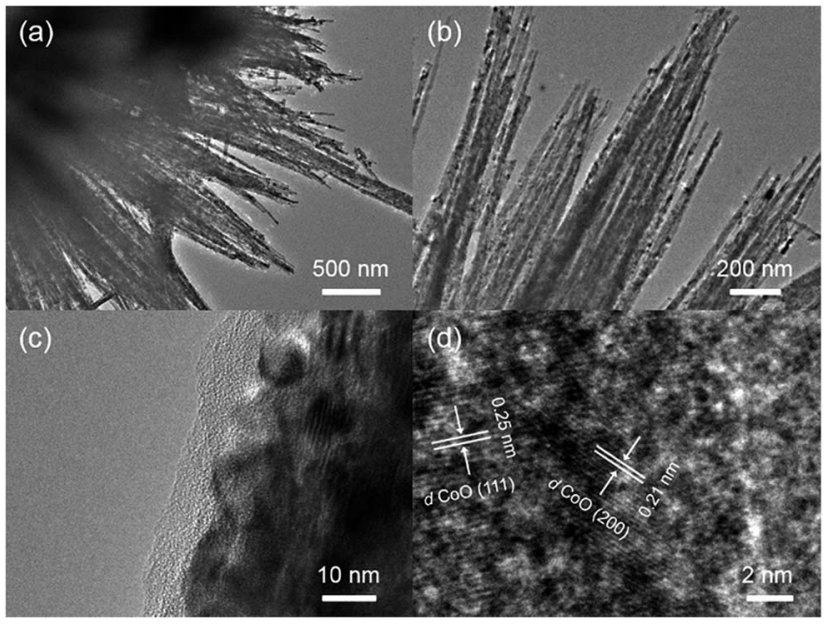

Fig. 2 ( $a$ and b) TEM images of the $\mathrm{CoO}-\mathrm{C}$ micro/nano hierarchical structure. (c) TEM image of the individual nanowire, exhibiting the porous structure of inner $\mathrm{CoO}$ and the thin carbon coating layer. (d) HRTEM image exhibiting the (200) and (111) lattice planes of CoO. 
weight loss is about $28.4 \%$ above $300{ }^{\circ} \mathrm{C}$, because of the decomposition of precursor into $\mathrm{CoO}, \mathrm{CO}_{2}$ and $\mathrm{H}_{2} \mathrm{O}$ in the $\mathrm{N}_{2}$ atmosphere. This value is lightly smaller than the theoretical value $(30.5 \%)$, which maybe due to partial loss of $\mathrm{H}_{2} \mathrm{O}$ in the precursor crystal structure or partial surface oxidation of CoO. To prove the product composition of EDS element mapping was carried out and clearly showed the existence of Co, O and $\mathrm{C}$, confirming the CoO-C configuration profile (Fig. 3). In addition, basing on the TG data of the CoO-C composite in nitrogen atmosphere (Fig. S5 $\mathrm{b}_{\dagger}$ ), the weight percentage of carbon component was less than $5 \%$. It indicated a thin carbon coating layer, which agreed with the SEM and TEM observations.

Subsequently, the product crystalline structures investigated by XRD at various synthesis stages clearly prove the successful fabrication of CoO-C. For the precursor, all the identified peaks can be assigned to pure orthorhombic $\mathrm{Co}\left(\mathrm{CO}_{3}\right)_{0.5}(\mathrm{OH}) \cdot 0.11 \mathrm{H}_{2} \mathrm{O}$ (JCPDS card no. 48-0083, space group: $P 22{ }_{1} 2$ ) (Fig. 4 a). ${ }^{25}$ For the XRD pattern of final composite material, five obvious diffraction peaks coinciding with the (111), (200), (220), (311) and (222) planes of standard spectrum of face-centered cubic CoO (JCPDS no. 78-0431, space group: Fm3m (225)) (Fig. 4b). ${ }^{15}$ The sharp peaks indicated high crystallinity and relatively large crystallite size, which agreed with above TEM results. ${ }^{26}$ Meanwhile, the broad peak around $20^{\circ}$ indicated the coating of amorphous carbon layer. ${ }^{16}$ As a comparison, the single $\mathrm{CoO}$ sample without carbon coating process showed a similar XRD pattern except for the broad peak corresponding to carbon (Fig. S6†). In addition, the Raman spectrum in the range of $50-1200 \mathrm{~cm}^{-1}$ showed two characteristic peaks of $\mathrm{CoO}$ at 190 and $517 \mathrm{~cm}^{-1}$ which could be attributed to the $\mathrm{F}_{2 \mathrm{~g}}$ mode. Meanwhile the other two peaks at 471 and $678 \mathrm{~cm}^{-1}$ corresponded to the $\mathrm{E}_{\mathrm{g}}$ and $\mathrm{A}_{1 \mathrm{~g}}$ modes, respectively. (Fig. $\mathrm{S} 7 \dagger$ ). ${ }^{9}$ To investigate the porous characteristic of the CoO-C sample, we show the $\mathrm{N}_{2}$ adsorptiondesorption isotherm and pore-size distributions in Fig. 4c and $\mathrm{d}$, respectively. According to the IUPAC classification, the shape of the hysteresis loop was ascribed to type H3. ${ }^{27}$ The average pore size was about $13 \mathrm{~nm}$, and the mesoporous urchin-like CoO-O showed a high BET surface area of $76 \mathrm{~m}^{2} \mathrm{~g}^{-1}$.
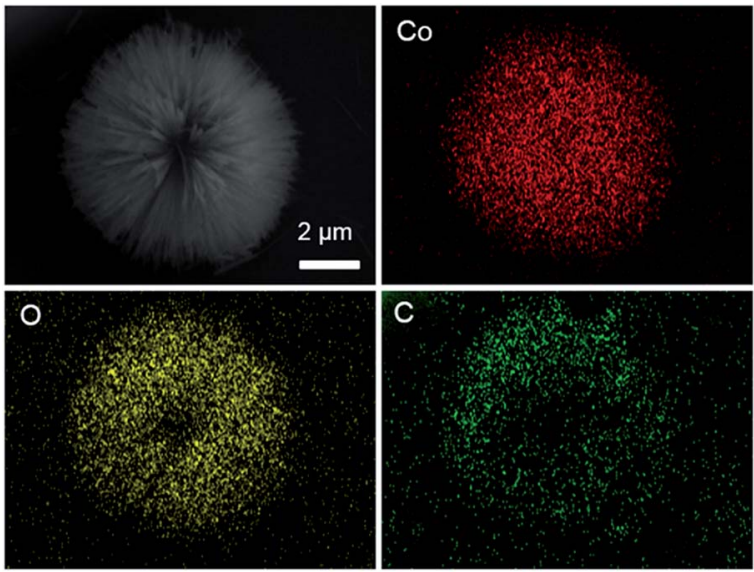

Fig. 3 EDS element mapping of the urchin-like $\mathrm{CoO}-\mathrm{C}$ microsphere, confirming the existence of $\mathrm{Co}, \mathrm{O}$ and $\mathrm{C}$.
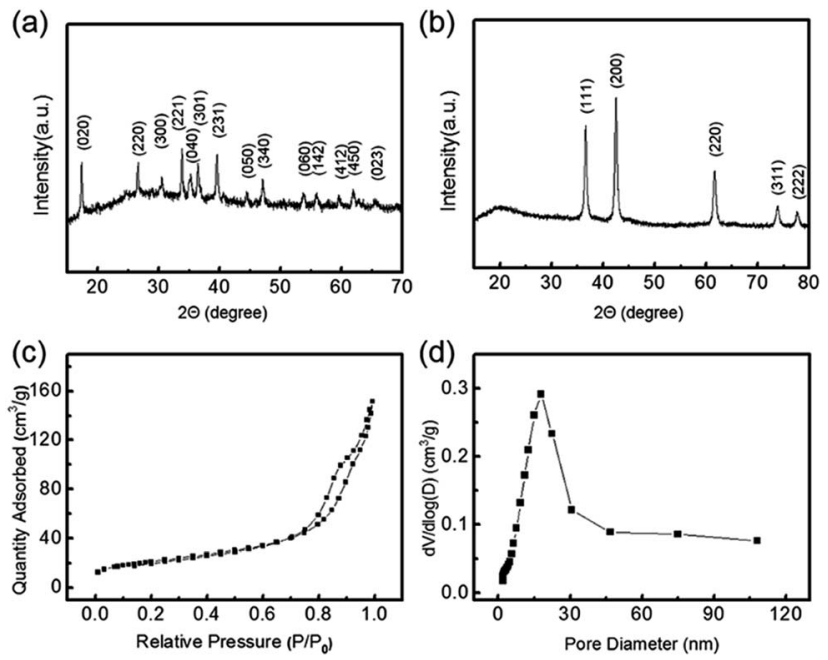

Fig. 4 (a and b) XRD patterns of the $\mathrm{Co}\left(\mathrm{CO}_{3}\right)_{0.5}(\mathrm{OH}) \cdot 0.11 \mathrm{H}_{2} \mathrm{O}$ precursor and $\mathrm{CoO}-\mathrm{C}$ composite material respectively. (c) $\mathrm{N}_{2}$ adsorption-desorption isotherm and (d) pore-size distribution curve of the urchin-like $\mathrm{CoO}-\mathrm{O}$.

Therefore, it was expected that the as-prepared mesoporous CoO-O microspheres would possess an excellent Li storage performance..$^{28}$

XPS characterization was used to further clarify chemical compositions. As shown in Fig. 5a, the full-survey spectrum clarified the presence of $\mathrm{Co}, \mathrm{O}$ and $\mathrm{C}$ elements, verifying the successful fabrication of CoO-C. For the Co $2 \mathrm{p}$ state, two characteristic peaks including the Co $2 \mathrm{p}_{3 / 2}$ states around $780.0 \mathrm{eV}$ and the Co $2 \mathrm{p}_{1 / 2}$ states around $795.5 \mathrm{eV}$ were observed, corresponding to the standard CoO composition (Fig. 5b). The presence of two prominent satellite peaks at 786.4 and $803.0 \mathrm{eV}$ further suggested the formation of the CoO phase after the calcination process in inert atmosphere. ${ }^{29}$ Fig. $5 \mathrm{c}$ displayed the $\mathrm{C}$ 1s peak, which corresponded to the carbon layers in the CoO-
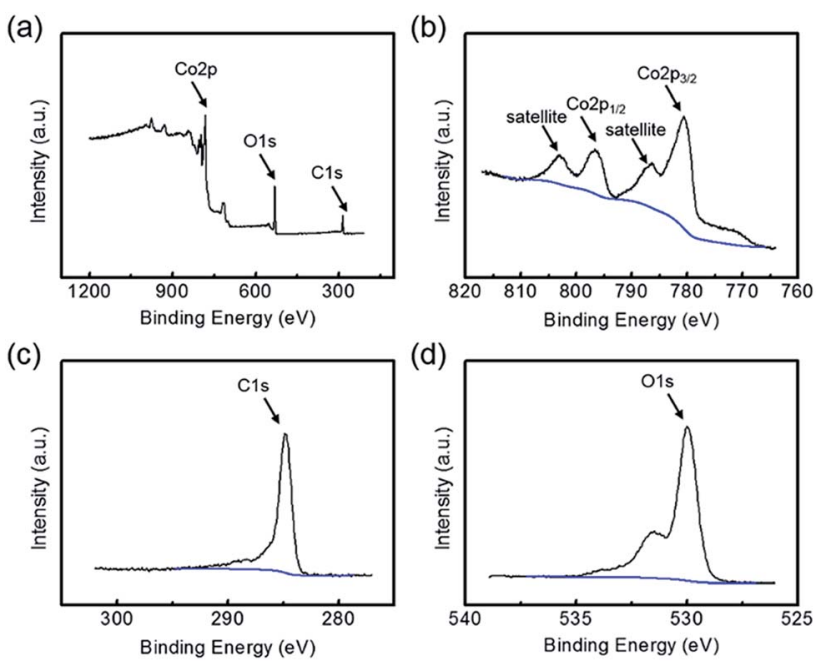

Fig. 5 (a) Full-survey, (b) Co 2p, (c) C 1s and (d) $O$ 1s states XPS characterizations of the $\mathrm{CoO}-\mathrm{O}$, further evidencing its chemical compositions. 
C. There were no obvious peaks existed around binding energies of 281 and $283 \mathrm{eV}$, indicating that carbon was not doped into the CoO phase..$^{15}$ For the $\mathrm{O} 1 \mathrm{~s}$ state, it was obvious that the O 1s XPS spectra can be deconvoluted into two sub-peaks (Fig. 5d). The peaks located at 530.0 was assigned to $\mathrm{O}$ bond in $\mathrm{CoO}$ crystalline networks, while the other smaller peaks around $531.8 \mathrm{eV}$ can be assigned to the weakly absorbed surface hydroxyl and structural water. ${ }^{30}$

\section{Lithium storage performances}

As-prepared urchin-like CoO-C micro/nano hierarchical structures were evaluated as LIB anode material to investigate its Listorage potential. Fig. 6a showed $1^{\text {st }}, 30^{\text {th }}$ and $50^{\text {th }}$ charge/ discharge voltage $v s$. capacity curves at a current density of $100 \mathrm{~mA} \mathrm{~g}^{-1}$ and a voltage range of $0.01-3.0 \mathrm{~V}\left(v s . \mathrm{Li} / \mathrm{Li}^{+}\right)$, which was consistent with the electrode composition units. A broad plateau starting around $0.8 \mathrm{~V}$ followed by a downward sloping was observed in the first discharge curve, which shifted to higher voltage and became less well-defined in the subsequent cycles. The plateau corresponded to the lithium storage in $\mathrm{CoO}$, and the slope can mainly be ascribed to the interaction of Co with the electrolyte to form SEI film. ${ }^{\mathbf{1 0 , 3 1}}$ The lower initial discharge plateau which differed from subsequent cycles could be attributed to the irreversible reactions and complex phase transformation of $\mathrm{CoO}$ to Co. ${ }^{7,32}$ The initial discharge and charge capacities was 1207 and $919 \mathrm{~mA} \mathrm{~h} \mathrm{~g}{ }^{-1}$. Being much higher than the theoretical capacity of $715 \mathrm{~mA} \mathrm{~h} \mathrm{~g}^{-1}$ for $\mathrm{CoO}$, the extra discharge capacity was mainly attributed to the electrolyte decomposition process which consumed $\mathrm{Li}^{+}$and formed the SEI film. ${ }^{33}$ Meanwhile, the irreversible capacity loss of $\sim 300$ $\mathrm{mA} \mathrm{h} \mathrm{g}^{-1}$ was attributed to the incomplete decomposition of SEI and $\mathrm{Li}_{2} \mathrm{O}$, accompanying with other factors including kinetic limitations. ${ }^{34}$ However, when compared with usual conversiontype anodes, the initial coulombic efficiency of $76 \%$ was relatively high, mainly owing to the carbon coating layer which introduce a stabilized electrode/electrolyte interface. ${ }^{\mathbf{1 2 , 1 3}}$ And well capacity retention was observed in subsequent cycles.

As this metal oxide-carbon synergistic system was expected to exhibit considerable performance advantages than singlecomposition metal oxide anode, therefore special capacity and cycling stability of the $\mathrm{CoO}-\mathrm{C}$ microspheres were comprehensively compared with the $\mathrm{CoO}$ with similar structure and without the carbon coating layer, in the voltage range of $0.01 \mathrm{~V}$ to $3.0 \mathrm{~V}$ versus $\mathrm{Li} / \mathrm{Li}^{+}$. Fig. $6 \mathrm{~b}$ highlighted that the introduction of carbon would decrease the special capacity in initial several cycles, however carbon could efficiently enhance the electrode capacity retention in subsequent cycles. The $\mathrm{CoO}-\mathrm{C}$ was able to maintain a discharge capacity as high as $755 \mathrm{~mA} \mathrm{~h} \mathrm{~g}^{-1}$, after 100 cycles at a current density of $100 \mathrm{~mA} \mathrm{~g}^{-1}$, indicating $80 \%$ retention of second discharge capacity. By contrast, that of the solely CoO was only $573 \mathrm{~mA} \mathrm{~h} \mathrm{~g}^{-1}$, with only $56 \%$ retention of second discharge capacity. In addition, the $\mathrm{CoO}-\mathrm{C}$ exhibited a coulombic efficiency of above $99 \%$ except for first several cycles, being slightly higher than that of the CoO electrode (Fig. S8a $\dagger$ ). When the current density was increased to $500 \mathrm{~mA}$ $\mathrm{g}^{-1}$, the CoO-C still maintained a discharge capacity of $667 \mathrm{~mA} \mathrm{~h} \mathrm{~g}^{-1}$ after 200 cycles with a high coulombic efficiency (Fig. 6c and S8b†). Meanwhile the solely $\mathrm{CoO}$ electrode underwent an intensified fading, and finally exhibited a discharge capacity less than half of the capacity of CoO-C. It was found that the advantage of carbon-coating treatment was more obvious in higher rate and longer-term cycles. The higher capacity retention of the $\mathrm{CoO}-\mathrm{C}$ can be attributed to the (a)

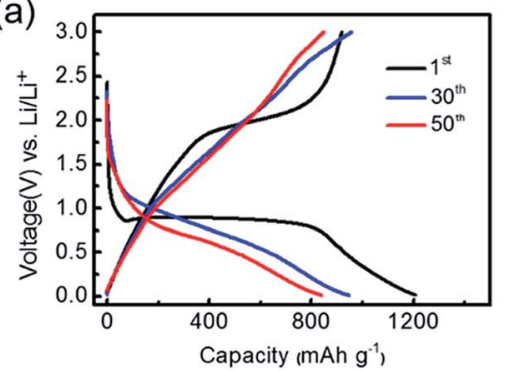

(d)

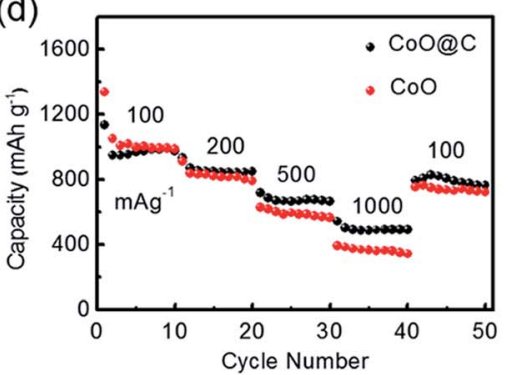

(b)

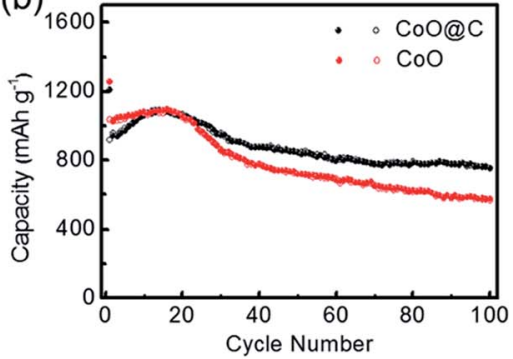

(e)

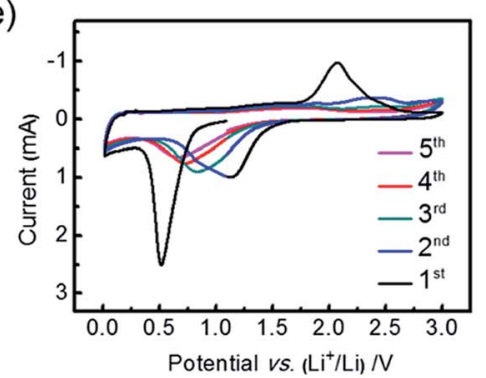

(c)

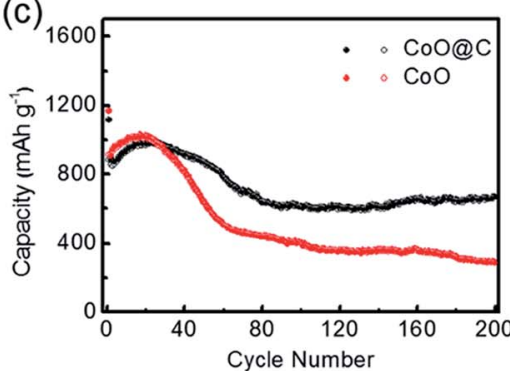

(f)

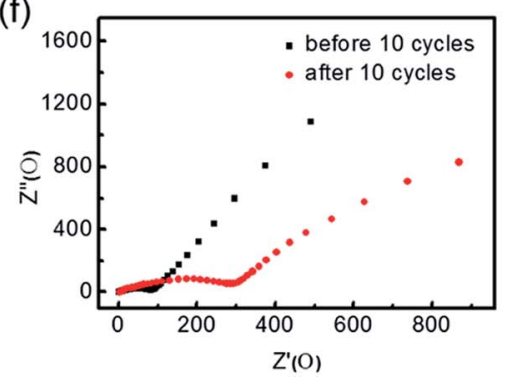

Fig. 6 (a) $1^{\text {st }}, 30^{\text {th }}$ and $50^{\text {th }}$ charge/discharge curves of the $\mathrm{CoO}-\mathrm{C}$ at a current density of $100 \mathrm{~mA} \mathrm{~g}^{-1}$. (b) Charge/discharge capacities vs. cycle numbers of $\mathrm{CoO}-\mathrm{C}$ and single $\mathrm{CoO}$ at a current density of $100 \mathrm{~mA} \mathrm{~g}^{-1}$. (c) Charge/discharge capacities vs. cycle numbers of two electrodes at a current density of $500 \mathrm{~mA} \mathrm{~g}^{-1}$. (d) Discharge capacities vs. cycle numbers of two electrodes at various current densities (mA $\mathrm{g}^{-1}$ ). (e) First 5 cycles of the $\mathrm{CV}$ curves for the $\mathrm{CoO}-\mathrm{C}$ at a scan rate of $0.1 \mathrm{mV} \mathrm{s}^{-1}$. (f) Impedance spectra of the CoO-C electrode before and after 10 cycles. 
fabrication of carbon coating layer on the $\mathrm{CoO}$ nanowires, which buffers the volume variation and enhances the electronic conduction.

Discharge capacities at various current densities were further compared. It was observed that capacities of both electrodes decreased monotonically as the rate increased, while the CoO-C exhibited a much higher capacity at higher rate (Fig. 6d). When tested at a high rate of $1000 \mathrm{~mA} \mathrm{~g}^{-1}$, the CoO-C microspheres were able to deliver a discharge capacity of nearly 500 $\mathrm{mA} \mathrm{h} \mathrm{g}^{-1}$. This means that the discharge or charge process could be finished in $30 \mathrm{~min}$, while a capacity over that of the commercial graphite was achieved. Moreover, when the rate turned back to $100 \mathrm{~mA} \mathrm{~g}^{-1}$ finally, the capacity could be retained at as high as more than $800 \mathrm{~mA} \mathrm{~h} \mathrm{~g}{ }^{-1}$. As a comparison, the solely $\mathrm{CoO}$ faced a more dramatic fade with increasing rates, exhibiting only $350 \mathrm{~mA} \mathrm{~h} \mathrm{~g}^{-1}$ at $1000 \mathrm{~mA} \mathrm{~g}^{-1}$.

In order to understand the electrochemical reactions during lithiation/delithiation process more explicitly, first 5 cycles of cyclic voltammetry (CV) curves for the CoO-C electrode were carried out, at a scan rate of $0.1 \mathrm{mV} \mathrm{s}^{-1}$ and a voltage range of 0.01-3.0 V (Fig. 6e). The profiles were in good agreement with the Li-storage mechanism for the CoO-based anodes: $\mathrm{CoO}+2 \mathrm{Li}^{+}$ $+2 \mathrm{e}^{-} \leftrightarrow \mathrm{Co}+\mathrm{Li}_{2} \mathrm{O} .^{35}$ There was a sharp peak at $0.5-0.6 \mathrm{~V}$ in the first cathodic scan, which corresponded to reduction of $\mathrm{CoO}$ to Co and irreversible reactions including the formation of the SEI film. ${ }^{36,37}$ From the second cycles, the peak had a positive shift to about $0.9 \mathrm{~V}$ and a decrease of density, which might be owed to the decreased surface energy of the CoO anode after the initial lithiation/delithiation process. ${ }^{14}$ In addition, cathodic peaks at lower than $0.1 \mathrm{~V}$ could be ascribed to the lithiation of carbon. ${ }^{33}$ Fig. 6f showed the Nyquist impedance plots of the $\mathrm{CoO}-\mathrm{C}$ electrode before and after 10 cycles, in which inclined lines at low frequency and depressed semicircles at high-medium frequency were ascribed to the lithium diffusion impedance and charge impedance respectively. ${ }^{18}$ It exhibited higher impedance after initial five cycles, which was mainly attributed to the formation of SEI film. ${ }^{8}$

Overall, owing to the synergistic effect from the carbon coating layer, it was observed that Li-storage performance of the original CoO could be enhanced to a great extent, especially at high rate condition. Compared with previous studies on cobalt oxide-based multiple or single component nanostructures (Table S1, ESI $\dagger$ ), this CoO-C composite electrode also exhibited considerable performance advantages. ${ }^{14,15,25,38-41}$ The strong potential of CoO-C micro/nano hierarchical structures was attributed to several factors: (1) CoO served as high capacity Listorage framework. (2) The nanowire basic unit provided rapid axial charge transferring and shortened diffusion path for both $\mathrm{Li}^{+}$and electrons which benefited the rate capability. (3) The 3D urchin-structure provided sufficient free space to buffer the volume variation which benefited the cycling stability. (4) The carbon coating layer complemented the low conductivity and volume variation issues for inner hetero-material, thus helped to obtain good capacity retention and superior performance at high rate. It also played a significant role like an artificial SEI layer to introduce stabilized electrode/electrolyte interface, without which inner active materials would be exhausted more intensively after long-term cycles. (5) At the multi-phase interface of this hetero-structure and $\mathrm{Co} / \mathrm{Li}_{2} \mathrm{O}$ interface formed after the conversion reaction, there existed possible interfacial lithium storage mechanism which further enhanced the system energy density. ${ }^{23}$

\section{Conclusions}

In summary, we have designed and constructed urchin-like CoO-C micro/nano hierarchical structures for efficient Listorage, via a sequential transformation route. This preparation strategy, based on the initial hydrothermal synthesis of urchin-like cobalt carbonate hydroxide precursor, subsequent glycosidation of glucose as carbon precursor and final calcination in inert atmosphere, provided the possibility of fabricating ideal hetero-nanostructures with targeted properties. CoO basic units and carbon coating layers were fabricated into a synergistic system, and the energy \& power densities of the composite $\mathrm{CoO}-\mathrm{C}$ were greatly improved comparing with the single $\mathrm{CoO}$, exhibiting a capacity as high as $755 \mathrm{~mA} \mathrm{~h}^{-1}$ at a rate of $100 \mathrm{~mA}$ $\mathrm{g}^{-1}$ as LIB anode material. We anticipate that this work may open a low-cost and well-established avenue for the large-scale production of high performance LIB anodes based on transition metal oxides.

\section{Acknowledgements}

This work was supported by National Natural Science Foundation of China (No. 21276257, 91534109 and 21503006), the "Strategic Priority Research Program" of the Chinese Academy of Sciences (No. XDA09010103), National Key Projects for Fundamental Research and Development of China (No. 2016YFB0100100), and the Research Foundation for Youth Scholars of Beijing Technology and Business University (no. QNJJ2014-14).

\section{References}

1 K. K. Sakimoto, A. B. Wong and P. D. Yang, Science, 2016, 351, 74.

2 M. R. Gao, J. X. Liang, Y. R. Zheng, Y. F. Xu, J. Jiang, Q. Gao, J. Li and S. H. Yu, Nat. Commun., 2015, 6, 5982.

3 Y. K. Sun, Z. H. Chen, H. J. Noh, D. J. Lee, H. G. Jung, Y. Ren, S. Wang, C. S. Yoon, S. T. Myung and K. Amine, Nat. Mater., 2012, 11, 942.

4 V. Aravindan, Y. S. Lee and S. Madhavi, Adv. Energy Mater., 2015, 5, 1402225.

5 J. Yu, S. M. Chen, W. J. Hao and S. J. Zhang, ACS Nano, 2016, 10, 2500.

6 S. L. Yang, C. Y. Cao, G. Li, Y. B. Sun, P. P. Huang, F. F. Wei and W. G. Song, Nano Res., 2015, 8, 1339.

7 K. Z. Cao, L. F. Jiao, Y. C. Liu, H. Q. Liu, Y. J. Wang and H. T. Yuan, Adv. Funct. Mater., 2015, 25, 1082.

8 C. Chae, K. W. Kim, S. J. Kim, D. Lee, Y. Jo, Y. J. Yun, J. Moon, Y. Choi, S. S. Lee, S. Choi and S. Jeong, Nanoscale, 2015, 7, 10368. 
9 W. Y. Yuan, M. Zhao, J. Yuan and C. M. Li, J. Power Sources, 2016, 319, 159.

10 D. D. Li, L. X. Ding, S. Q. Wang, D. D. Cai and H. H. Wang, J. Mater. Chem. A, 2014, 2, 5625.

11 X. L. Zhou, Y. R. Zhong, M. Yang, Q. Zhang, J. P. Wei and Z. Zhou, ACS Appl. Mater. Interfaces, 2015, 7, 12022.

12 Y. Wang, H. J. Zhang, L. Lu, L. P. Stubbs, C. C. Wong and J. Y. Lin, ACS Nano, 2010, 4, 4753.

13 J. S. Cho, Y. J. Hong and Y. C. Kang, ACS Nano, 2015, 9, 4026.

14 K. W. Xie, P. Wu, Y. Y. Zhou, Y. Ye, H. Wang, Y. W. Tang, Y. M. Zhou and T. H. Lu, ACS Appl. Mater. Interfaces, 2014, 6, 10602.

15 F. F. Wu, X. J. Ma, J. K. Feng, Y. T. Qian and S. L. Xiong, J. Mater. Chem. A, 2014, 2, 11597.

16 N. Jayaprakash, W. D. Jones, S. S. Moganty and L. A. Archer, J. Power Sources, 2012, 200, 53.

17 X. W. Lou, D. Deng, J. Y. Lee and L. A. Archer, Chem. Mater., 2008, 20, 6562 .

18 W. J. Hao, S. M. Chen, Y. J. Cai, L. Zhang, Z. X. Li and S. J. Zhang, J. Mater. Chem. A, 2014, 2, 13801.

19 H. J. Qiu, L. Liu, Y. P. Mu, H. J. Zhang and Y. Wang, Nano Res., 2015, 8, 321.

20 W. Y. Li, L. N. Xu and J. Chen, Adv. Funct. Mater., 2005, 15, 851.

21 B. Jang, M. Park, O. B. Chae, S. Park, Y. Kim, S. M. Oh, Y. Piao and T. Hyeon, J. Am. Chem. Soc., 2012, 134, 15010.

22 Y. C. Jiao, D. D. Han, L. M. Liu, L. Ji, G. N. Guo, J. H. Hu, D. Yang and A. G. Dong, Angew. Chem., Int. Ed., 2015, 54, 5727.

23 Y. F. Zhukovskii, P. Balaya, E. A. Kotomin and J. Maier, Phys. Rev. Lett., 2006, 96, 058302.

24 H. Wang, C. Qing, J. L. Guo, A. A. Aref, D. M. Sun, B. X. Wang and Y. W. Tang, J. Mater. Chem. A, 2014, 2, 11776.
25 Y. Wang, H. Xia, L. Lu and J. Lin, ACS Nano, 2010, 4, 1425. 26 J. S. Cho, Y. J. Hong and Y. C. Kang, ACS Nano, 2015, 9, 4026. 27 D. Q. Liu, X. Wang, X. B. Wang, W. Tian, Y. Bando and D. Golberg, Sci. Rep., 2013, 3, 2543.

28 Z. Wang, W. Jia, M. L. Jiang, C. Chen and Y. D. Li, Nano Res., 2016, 9, 2026.

29 S. A. Needham, G. X. Wang, K. Konstantinov, Y. Tournayre, Z. Lao and H. K. Liu, Electrochem. Solid-State Lett., 2006, 9, A315.

30 J. K. Chang, C. M. Wu and I. W. Sun, J. Mater. Chem., 2010, 20, 3729.

31 A. K. Rai, L. T. Anh, J. Gim and J. Kim, Ceram. Int., 2013, 39, 9325.

32 F. D. Wu and Y. Wang, J. Mater. Chem., 2011, 21, 6636.

33 L. Yang, S. Cheng, Y. Ding, X. B. Zhu, Z. L. Wang and M. L. Liu, Nano Lett., 2012, 12, 321.

34 M. V. Reddy, T. Yu, C. H. Sow, Z. X. Shen, C. T. Lim, G. V. S. Rao and B. V. R. Chowdari, Adv. Funct. Mater., 2007, 17, 2792.

35 P. Poizot, S. Laruelle, L. Dupont and J. M. Tarascon, Nature, 2000, 407, 496.

36 Y. Qi, H. Zhang, N. Du and D. Yang, J. Mater. Chem. A, 2013, 1, 2337.

37 M. V. Reddy, G. Prithvi, K. Loh and B. V. R. Chowdari, ACS Appl. Mater. Interfaces, 2014, 6, 680.

38 Y. G. Li, B. Tan and Y. Y. Wu, Nano Lett., 2008, 8, 265.

39 X. Wang, X. L. Wu, Y. G. Guo, Y. T. Zhong, X. Q. Cao, Y. Ma and J. N. Yao, Adv. Funct. Mater., 2010, 20, 1680.

40 S. L. Xiong, J. S. Chen, X. W. Lou and H. C. Zeng, Adv. Funct. Mater., 2012, 22, 861.

41 Y. C. Qiu, S. H. Yang, H. Deng, L. M. Jin and W. S. Li, J. Mater. Chem., 2010, 20, 4439. 